\title{
A UTILIZAÇÃO DE UM SOFTWARE LIVRE PARA DETERMINAÇÃO DOS ESFORÇOS INTERNOS DE CARGAS MÓVEIS EM VIGAS DE PONTES DE CONCRETO
}

\author{
F. J. R. MASCARENHAS ${ }^{1}$, A. L. CHRISTOFORO², R. C. CARVALHO \\ Universidade Federal de São Carlos \\ fer.jr.resende@hotmail.com ${ }^{1}$
}

Submetido 27/01/2020 - Aceito 02/04/2020

DOI: $10.15628 /$ holos.2020.9466

\section{RESUMO}

O Ftool é utilizado na engenharia civil pelas facilidades e simplificações na modelagem estrutural; por não demandar computadores robustos para sua utilização; não ter tempo de processamento elevado nas análises, sendo geralmente rápidas e por ser um software gratuito. Ele permite criar cargas móveis e essas são usadas nos cálculos das envoltórias dos esforços internos em vigas de pontes. Apesar disso, significativo número de trabalhos, dissertações e teses, na área de pontes, sobretudo dos cursos de pós-graduação, ainda não o utilizam nas análises das cargas móveis em vigas de pontes. Nesse sentido, este trabalho objetiva fomentar o uso do Ftool para se realizar análises estruturais de vigas de pontes em vigas, seja na determinação das respectivas linhas de influência ou dos esforços internos; para que estudantes de pós-graduação e pesquisadores passem a utilizá-lo em seus respectivos trabalhos. São desenvolvidos quatro exemplos numéricos e em cada um emprega-se um software pago, SAP2000, Ansys ou GAP-GEL v1, e os resultados são comparados com o Ftool. Em todos os exemplos, as variações dos resultados são inferiores a $9 \%$, no pior dos casos, mas na maioria deles, essa variação é inferior a $3 \%$. Essas diferenças podem ser tidas como aceitáveis, pois, posteriormente, são aplicados diferentes coeficientes de majoração. Por fim, o Ftool mostrou-se eficaz e robusto, com resultados confiáveis e, portanto, recomendado para a determinação dos diferentes esforços internos e TTL nas vigas, devido a diferentes cargas móveis atuantes em tabuleiros de pontes compostas por duas, três, sete e oito vigas. Sem que haja a necessidade de se empregar software mais complexos e pagos para se realizar tais análises.

PALAVRAS-CHAVE: Ftool, Análise Estrutural, Esforços Internos, Vigas, Pontes.

\section{THE USE OF A FREE SOFTWARE FOR DETERMINATION OF THE INTERNAL FORCES OF LIVE LOADS IN BEAMS OF CONCRETE BRIDGES}

\begin{abstract}
Ftool is used in civil engineering due to its facilities and simplifications in structural modeling; for not requiring robust computers for its use; not having high processing time in the analyzes, being generally fast and for being a free software. It allows the creation of live loads, and these are used in calculating the envelope of internal forces in bridge beams. Despite this, a significant number of projects, dissertations and theses in the area of bridges, especially in graduate courses, still do not use it in the analysis of life loads in bridge beams. In this sense, this project aims to encourage the use of Ftool to carry out structural analysis in beams of bridge beams, whether in determining the respective lines of influence or internal efforts; so that graduate students and researchers start to use it in their respective projects.
\end{abstract}

Four numerical examples are developed and each one uses paid software, SAP2000, Ansys or GAP-GEL v1, and the results are compared with Ftool. In all examples, the variations in the results are less than $9 \%$, in the worst case, but in most of them, this variation is less than $3 \%$. These differences can be considered as acceptable, since, later, different coefficients are applied. Finally, the Ftool proved to be effective and robust, with reliable results and, therefore, recommended for the determination of the different internal and TTL efforts in the beams, due to different live loads acting on bridge decks composed of two, three, seven and eight beams. Without the need to use more complex and paid software to carry out such analyzes.

KEYWORDS: Ftool, Structural Analysis, Internal Forces, Beams, Bridges. 


\section{INTRODUÇÃO}

Desde o advento dos softwares e demais recursos computacionais e posterior utilização deles na engenharia civil, com destaque para a engenharia de estruturas, o tempo requerido para a análise estrutural e o dimensionamento das diferentes estruturas têm reduzido, bem como temse dimensionado estruturas cada vez mais complexas.

A área de engenharia de pontes e viadutos é uma das que se beneficiou e avançou consideravelmente com a introdução de programas de computador para a análise estrutural e o dimensionamento.

Ao se realizar o projeto de pontes e viadutos, assim como a recuperação estrutural dos mesmos, os engenheiros devem considerar todas as possíveis ações que exercerão influências em seus desempenhos estruturais. Dentre essas, as cargas móveis merecem atenção especial pela complexidade de análise que elas apresentam. Nesse sentido, as envoltórias de esforços cortantes e momentos fletores oriundas dessas cargas são relevantes para se dimensionar e/ou verificar os elementos estruturais de pontes e viadutos.

Abrishami (2017, p. 7-12), professor do Departamento de Engenharia Civil da Universidade de Toronto, esclarece que, atualmente, a análise estrutural de pontes e viadutos "é geralmente conduzida por meio de softwares para aproveitar as habilidades computacionais dos computadores". Os softwares mais empregados na análise estrutural e dimensionamento dessas estruturas são o SAP2000, RISA 3D, MIDAS Civil, ANSYS e Abaqus FEA, todos de acesso não gratuito e que requerem conhecimentos mais aprofundados para se utilizá-los (ABRUSHAMI, 2017).

Apesar da existência de diversos softwares empregados no dimensionamento de pontes e viadutos, eles são pagos em sua maioria e muitas vezes requerem conhecimentos mais específicos para serem utilizados. Além disso, eles geralmente apresentam grande custo computacional e de tempo para realizar as análises. Tais fatos acabam por inviabilizar o emprego deles nos âmbitos acadêmico, científico e técnico na Engenharia Civil.

Silva e Stoeterau (2016), da Universidade de São Paulo, esclarecem que a etapa de préprocessamento de softwares baseados nos métodos de elementos finitos é aquela que requer maior esforço de quem utiliza, seja no estabelecimento da geometria do modelo, das propriedades dos materiais, na geração da malha, aplicação das condições de contorno, função de força, e, por fim, a validação do modelo. Segundo os autores, essa última etapa é muito complexa "e exige um esforço enorme do analista e como a solução de modelos complexos é algo de demanda uma grande quantidade de tempo, é importante verificar se o modelo foi construído de forma correta" (SILVA, STOETERAU, 2016, p. 10).

Um software que tem sido amplamente usado por discentes, docentes e pesquisadores na Engenharia Civil é o Ftool (Two-dimensional Frame Analysis Tool). Dentre os motivos de sua utilização citam-se: as facilidades e simplificações na modelagem estrutural; não demandar computadores robustos para sua utilização; não ter tempo de processamento elevado nas análises, sendo geralmente rápidas; e, sobretudo, por ser um software de acesso livre e gratuito. 
Dentre as diferentes funcionalidades do Ftool, assinala-se que ele "permite que sejam criadas cargas móveis que são usadas nos cálculos de envoltórias de esforços internos" (MARTHA, 2018 , p. 30). Mesmo ele apresentando essas características de utilização, significativo número de trabalhos, dissertações e teses oriundos das universidades brasileiras, na área de pontes e viadutos, sobretudo dos cursos de pós-graduação, ainda não o utilizam para as análises das cargas móveis em vigas de pontes.

Nesse sentido, este trabalho objetiva fomentar a utilização do Ftool para se realizar análises estruturais de vigas de pontes e/ou viadutos em vigas, seja na determinação das respectivas linhas de influência ou dos esforços internos. Com o intuito de que estudantes de pós-graduação, professores e/ou pesquisadores passem a utilizar o Ftool em seus respectivos trabalhos.

Baseado nisso, são apresentados quatros exemplos numéricos de diferentes autores. Nestes exemplos, analisa-se os resultados dos esforços cortantes e momentos fletores oriundos de cargas móveis em vigas de pontes de concreto, com diferentes quantidades de vigas. Os resultados encontrados através do uso de softwares de elementos finitos comerciais, com modelagem 3D, são comparados com aqueles obtidos pelo Ftool, com modelagem 2D, versão básica 4.00 .

O intuito da análise e da comparação é evidenciar que o Ftool é uma ferramenta recomenda e que pode ser utilizada para o estudo e análise estrutural de vigas de pontes de concreto com diferente número de vigas. Pois, apesar da existência dos diversos softwares pagos, Carvalho Neto e Veloso $(2014$, p. 2) pontuam que no caso da determinação dos momentos fletores de cargas móveis, essas ferramentas computacionais "levaram a um considerável aumento no tempo de processamento e de esforço computacional".

Para tanto, apresenta-se, primeiramente, uma revisão bibliográfica sobre a definição de pontes e viadutos, bem como a relevância de tais estruturas para o sistema de transportes. Após discorrido sobre as principais informações do software, parte-se para a apresentação dos trabalhos encontrados na literatura, demonstrando-se os diversificados propósitos de utilização do Ftool. Por fim, são desenvolvidos os quatro exemplos numéricos.

\subsection{Pontes e viadutos}

A Norma Brasileira (NBR), da Associação Brasileira de Normas Técnicas (ANBT), 7188:2013, de cargas móveis em pontes, viadutos e passarelas define ponte como a "estrutura sujeita a ação de carga em movimento, com posicionamento variável (chamada de carga móvel), utilizada para transpor um obstáculo natural (rio, córrego, vale, etc.)" e viaduto como a "estrutura para transpor um obstáculo artificial (avenida, rodovia, etc.)" (ABNT, 2013, p. 1).

Mascarenhas, Carvalho e Vitório (2019, p. 4) esclarecem que apesar das 8.336 pontes e viadutos cadastradas pelo Departamento Nacional de Infraestrutura de Transportes (DNIT), "é sabido que existe um número bem maior de pontes em rodovias estaduais, municipais e sob concessão que não constam desse levantamento e que podem chegar a 120 mil de acordo com dados não oficiais". 
Sabe-se que pontes e viadutos são elementos indispensáveis e decisivos "nos desenvolvimentos sociais e econômicos de cidades", uma vez que as rodovias garantem a circulação de bens, subsídios para produção e os frutos dessa produção, além de permitir a "livre movimentação de pessoas" (MILANI; KRIPKA, 2012, p. 26).

\section{FTOOL}

Idealizado pelo professor Luiz Fernando Martha, do Departamento de Engenharia Civil da PUC-Rio, o software fora desenvolvido inicialmente "com um enfoque educacional", o Ftool "evoluiu para uma ferramenta frequentemente utilizada inclusive em projetos executivos de estruturas profissionais" (FTOOL, 2019). O software possui uma versão avançada, a qual é paga e a versão básica de acesso gratuito. A versão básica:

Permite que o usuário defina modelos de forma eficiente e simples. O Ftool analisa um modelo estrutural fornecendo tanto resultados simples, como diagramas de esforços internos e deformadas, quanto de linhas de influência em qualquer ponto da estrutura e envoltórias de esforços para trens-tipo. Seções transversais podem ser definidas de forma paramétrica de acordo com diversos templates (retangular, seção T, L, I, etc.), selecionando seções tabeladas de diversas entidades (Gerdau, AISC, etc.), ou de forma genérica (definindo as propriedades geométricas como área e momento de inércia). Membros estruturais podem ser calculados pelas teorias de EulerBernouilli ou Timoshenko. Apoios podem ser rígidos ou elásticos e podem ser rotacionados, ou aceitar deslocamentos impostos. Isso permite que diversos tipos de estruturas, das mais simples às mais complexas, possam ser modeladas no Ftool em poucos minutos (FTOOL, 2019).

A seguir, mostra-se a figura 1 na qual se apresenta a tela de entrada (principal) do Ftool, na qual as modelagens das estruturas são realizadas.

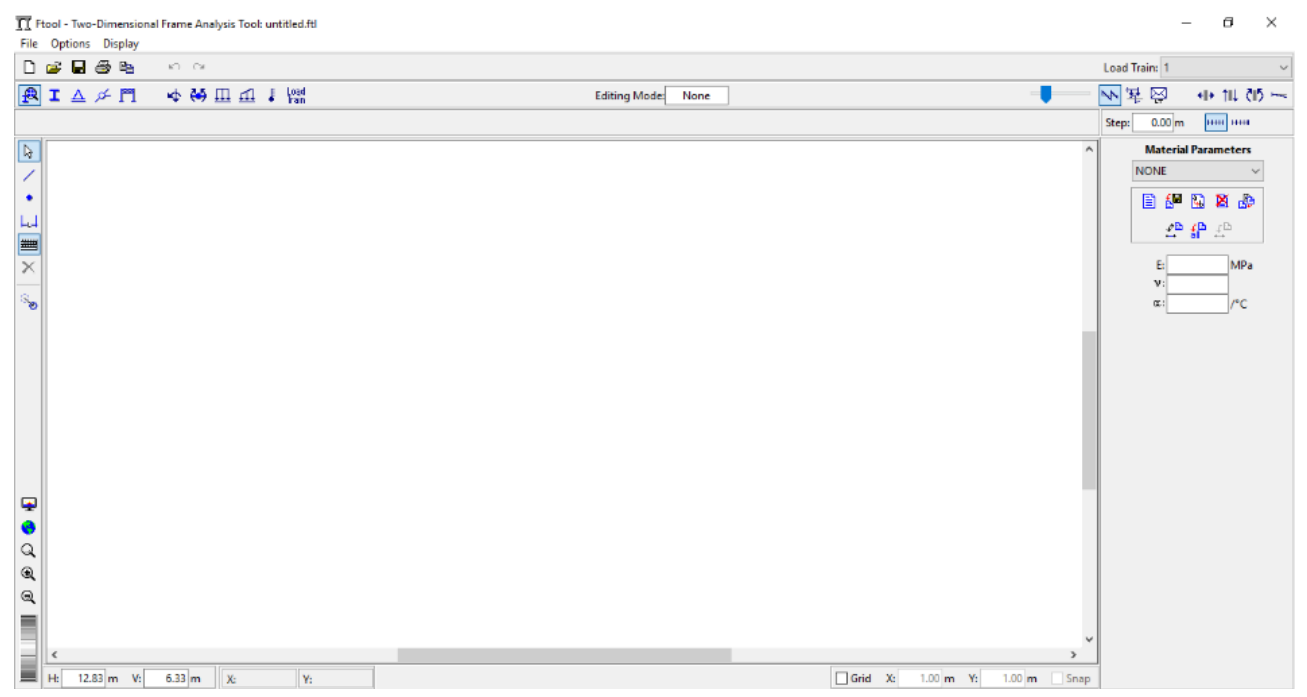

Figura 1: Tela principal do Ftool (FTOOL (2018))

Na determinação das envoltórias de momentos fletores, esforços axiais e cortantes de cargas móveis, o Ftool utiliza o princípio das linhas de influência, que é um modelo simplificado já consagrado que pode ser visto em diversificadas publicações, tais como Carvalho (2017). A figura 
2 mostra um exemplo de linhas de influência para uma ponte teórica de seção transversal de 12,00 metros $(m)$, usando as cargas concentradas do trem-tipo normativo brasileiro, TB 450 (ABNT, 2013).

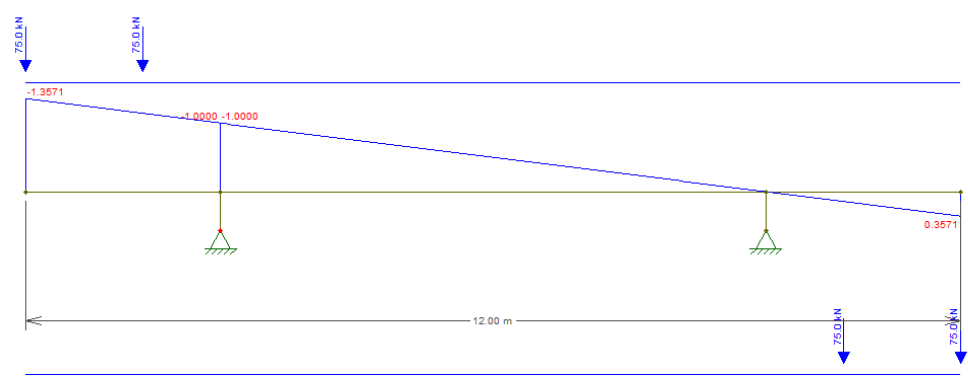

Figura 2: Exemplo de linha de influência (PRÓPRIOS AUTORES (2019))

A escolha pela utilização do Ftool deve-se ao fato dele ser uma ferramenta gratuita e por ser empregado em trabalhos técnicos e no meio acadêmico, sobretudo como ferramenta pedagógica. Nessa perspectiva, citam-se alguns exemplos de textos acadêmicos e documentos técnicos nos quais se empregou o Ftool para se realizar as análises estruturais.

Mascarenhas, Dias e Bonaldo (2016) investigaram sobre a utilização de softwares por estudantes da disciplina de teoria das estruturas, do curso de graduação em engenharia civil, da PUC Minas, unidade Barreiro. Os autores constataram que $64,03 \%$ dos discentes conheciam o Ftool. Passos et al. (2016) realizaram um mapeamento sistemático acerca dos softwares mais utilizados nos cursos de engenharia civil no Brasil e concluíram que os 5 mais utilizados são, em ordem decrescente de utilização: Matlab, Excel, Scilab, Ftool e GDFácil.

Barros et al. (2018) e Santos Jr. et al. (2018) evidenciam o emprego do Ftool por docentes e discentes de Engenharia Civil nas disciplinas de mecânica dos materiais, resistência dos materiais e estática das estruturas. E todos os autores optaram pelo Ftool pelo mesmo ser gratuito, apresentar resultados confiáveis e possuir modelagem simples.

No que se refere à utilização do Ftool para cargas móveis, menciona-se o relatório técnico elaborado pela concessionária de rodovias Rota do Oeste S.A. emitido para a Agência Nacional de Transportes Terrestres (ANTT). No relatório, realizou-se a avaliação dos impactos oriundos das cargas dos veículos reais que "trafegam nas OAEs [pontes e viadutos] nos elementos portantes principais de suas estruturas, para quantificação dos efeitos da redução da vida útil" foi realizada (ROTA DO OESTE S.A., 2016, p. 7). A determinação das envoltórias de momentos fletores das cargas móveis nas estruturas de concreto armado analisadas deu-se por meio do Ftool.

Destaca-se, também, o trabalho de doutorado desenvolvido por Baroni (2010). Em seus estudos a autora empregou o Ftool na determinação dos momentos fletores atuantes nas pontes por ela analisadas. A autora analisou pontes de concreto armado com 2 vigas longarinas biapoiadas com comprimentos de 10,00, 15,00 e 20,00 m e seção transversal de 8,00 e 13,00 m.

Mencionam-se também os trabalhos de Cruz Santos (2016) e Turmina (2016) que empregaram o Ftool em suas respectivas dissertações de graduação em Engenharia Civil para determinação dos momentos fletores devido às cargas móveis nas pontes por eles analisadas. 
Medeiros (2016) e Moura et al. (2016), em seus respectivos trabalhos (artigos científicos), utilizaram o Ftool como ferramenta para determinação das envoltórias de momentos fletores das cargas móveis de pontes com vigas múltiplas, sendo que cada trabalho utilizou abordagens e hipóteses de cálculo diferentes no Ftool, mas todas baseadas em linhas de influência.

Cita-se, ainda, o trabalho de Drun e Souza (2018) no qual foi empregado o Ftool, com isso, "a análise concentrou-se na obtenção do momento fletor atuante em uma ponte fictícia" utilizando-se as cargas móveis normativas e de veículos reais que trafegam nas rodovias brasileiras.

Por fim, menciona-se o trabalho desenvolvido por Mascarenhas e Chust Carvalho (2019, p. 303) que analisam "a vida útil à fadiga da armadura longitudinal em vigas de pontes de concreto armado considerando-se o número real de veículos pesados de 2 a 6 eixos em um trecho rodoviário do estado de São Paulo, Brasil". Para tanto, os autores empregaram o Ftool para determinação das envoltórias de momento fletor das cargas móveis nas pontes por eles analisadas.

Apesar do estado da arte apresentado acima acerca da utilização do Ftool, o software ainda não é opção primeira de estudantes e pesquisadores de pós-graduação em Engenharia Civil no Brasil. Dentre as hipóteses para não utilização pode-se mencionar o fato dele ser visto por algumas pessoas como um software com caráter mais pedagógico do que propriamente científico. Além disso, cita-se, também, a evolução e o aprimoramento dos softwares de elementos finitos, com diversas aplicabilidades nas Engenharias Civil e Estrutural, permitindo análises mais robustas.

Todavia, o emprego de softwares pagos de elementos finitos com fins pedagógicos, e até mesmo no âmbito de pesquisas (meio científico) que tratem e/ou estudem de pontes e viadutos, pode, por muitas vezes, limitar as pesquisas, seja pelo fato de serem softwares pagos, seja pela complexidade de utilização dos mesmos, ou pelo tempo de processamento dos modelos.

Carvalho Neto e Veloso (2014, p. 1) explicam que apesar dos "softwares comerciais de Elementos Finitos apresentam interfaces cada vez mais práticas e de fácil manuseio", eles acabam gerando um aumento no tempo de processamento do modelo analisado.

Nessa direção, este trabalho tem como foco principal evidenciar a aplicabilidade, robustez e confiabilidade do Ftool nas análises estruturais de cargas móveis em vigas de pontes com diferentes números de vigas, tendo em vista a determinação das cargas que compõem o trem tipo longitudinal máximo (TTL), dos esforços cortes e das envoltórias de momentos fletores de vigas de pontes e/ou viadutos. Demonstrando, além disso, que análises e pesquisas podem ser realizadas sem a necessidade de se empregar softwares mais complexos e/ou pagos.

\section{EXEMPLO NUMÉRICO 01}

O exemplo numérico 01 é baseado na ponte estudada por Albuquerque (2012). Em seu trabalho, o autor determinou os momentos fletores devido às cargas móveis de alguns veículos reais de carga que trafegam nas rodovias brasileiras no meio do vão da viga de uma ponte de concreto armado de vão de 10,00 m composta por duas vigas, com extensão de 12,80 m. 0 concreto utilizado tem $f_{c k}$ de $25 \mathrm{MPa}$. A figura 3 demonstra a seção transversal da ponte analisada. 


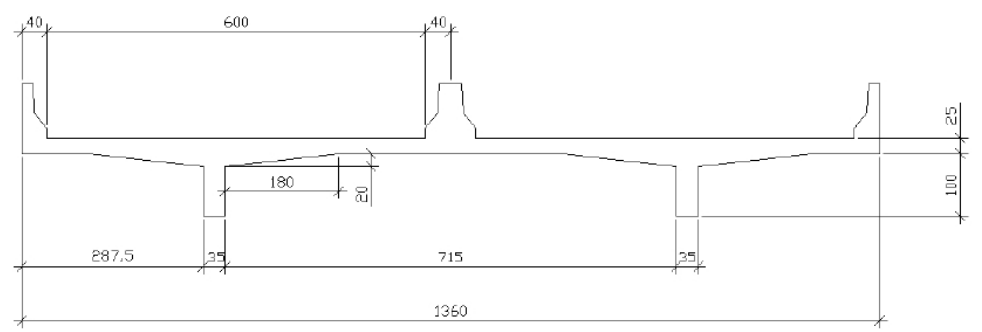

Figura 3: Seção transversal da ponte de Albuquerque (ALBUQUERQUE (2012))

Para tanto, o autor utilizou o software de elementos finitos SAP 2000, versão 14, Structural Analysis Program. Com isso, a referida ponte é modelada no Ftool (2D) versão básica 4.00 e os resultados encontrados são comparados com aqueles obtidos por Albuquerque (2012). A figura 4 demonstra o modelo estrutural 3D da ponte analisada realizado por Albuquerque (2012).

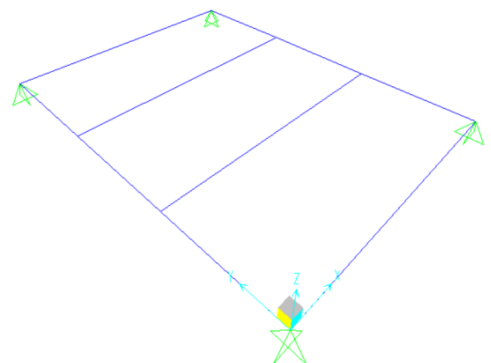

Figura 4: Modelo estrutural (ALBUQUERQUE (2012))

Nesse trabalho, são adotadas as seguintes considerações e hipóteses de cálculo no modelo de cargas móveis dos veículos reais a ser utilizado na verificação utilizando o Ftool, sendo elas as mesmas empregadas por Albuquerque (2012):

a) Albuquerque (2012) determinou os momentos fletores nas vigas devido à passagem de alguns veículos reais denominados $3 \mathrm{C}, \mathrm{O} 2 \mathrm{C}, \mathrm{O} 3 \mathrm{C}, 2 \mathrm{~S} 2$ e $2 \mathrm{~S} 3$, com diferentes pesos totais, conforme figura 5;
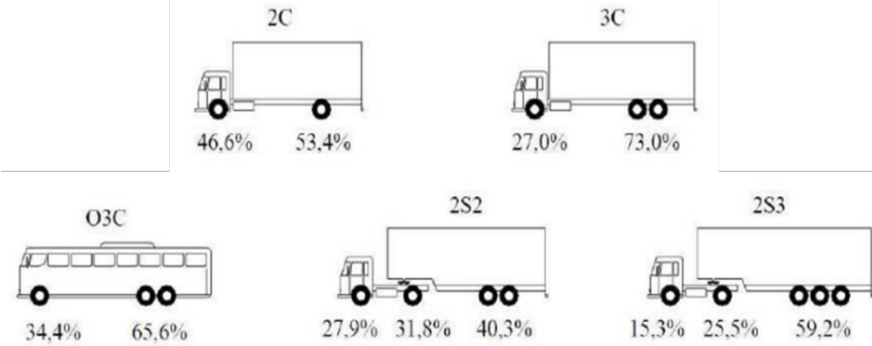

Figura 5: Veículo utilizados (ALBUQUERQUE (2012))

b) Transversalmente os veículos terão as mesmas dimensões, 1,90 m;

c) $100 \%$ dos veículos estarão posicionados no centro da faixa de tráfego, como a figura 6 ; 


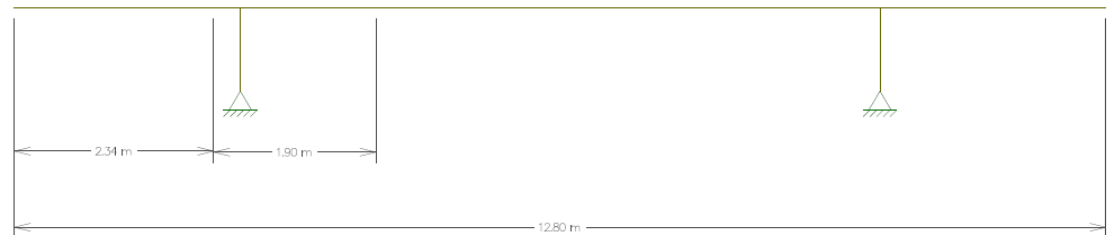

Figura 6: Posição transversal adotada para os veículos de carga (PRÓPRIOS AUTORES (2019))

d) Para determinação dos momentos fletores longitudinalmente na viga estudada usa-se a linha de influência de reação de apoio, determinada no Ftool, considerando-se os veículos na posição indicada no item (c). Feito isso chega-se ao conjunto de cargas chamados de trem-tipo longitudinal (TTL), com o qual os esforços de momento fletor das vigas serão determinadas.

\subsection{Análise e discussão dos resultados do Exemplo Numérico 01}

Os resultados para ambas as análises se encontram nas tabelas seguintes. Elas contêm as cargas totais para os diferentes tipos de veículos, os momentos calculados pelo SAP2000 (1) e pelo Ftool (2) e a variação encontrada entre ambos os resultados. As porcentagens negativas indicam que os valores de momentos fletores determinados pelo Ftool são maiores que os encontrados pelo SAP2000, já as porcentagens positivas indicam que os valores encontrados Ftool são menores.

Tabela 1: Comparação entre os momentos fletores no meio do vão: SAP2000 (1) e Ftool (2) - Veículo 2C

\begin{tabular}{c|c|c|c}
\hline Carga total $(\mathrm{kN})$ & SAP2000 $(\mathrm{kN} . \mathrm{m})$ & Ftool $(\mathrm{kN} . \mathrm{m})$ & Variação entre os esforços por (1) e (2) (\%) \\
\hline 22,3 & 43,6 & 42,6 & 2,29 \\
164 & 320,6 & 312,0 & 2,68 \\
195 & 381,2 & 371,0 & 2,68 \\
263 & 514,2 & 504,9 & 1,81 \\
386 & 754,7 & 734,6 & 2,66 \\
\hline
\end{tabular}

Fonte: (PRÓPRIOS AUTORES (2020))

Tabela 2: Comparação entre os momentos fletores no meio do vão: SAP2000 (1) e Ftool (2) - Veículo 3C

\begin{tabular}{c|c|c|c}
\hline Carga total $(\mathrm{kN})$ & SAP2000 (kN.m) & Ftool $(\mathrm{kN} . \mathrm{m})$ & Variação entre os esforços por (1) e (2) (\%) \\
\hline 22,3 & 43,0 & 42,8 & 0,47 \\
158 & 304,7 & 304,3 & 0,13 \\
236 & 455,1 & 454,7 & 0,09 \\
332 & 640,3 & 639,9 & 0,06 \\
446 & 860,1 & 859,1 & 0,12 \\
\hline
\end{tabular}

Fonte: (PRÓPRIOS AUTORES (2020))

Tabela 3: Comparação entre os momentos fletores no meio do vão: SAP2000 (1) e Ftool (2) - Veículo O3C

\begin{tabular}{c|c|c|c}
\hline Carga total $(\mathrm{kN})$ & SAP2000 (kN.m) & Ftool (kN.m) & Variação entre os esforços por (1) e (2) (\%) \\
\hline 22,3 & 38,3 & 38,8 & $-1,31$ \\
158 & 271,1 & 273,7 & $-0,96$ \\
229 & 392,9 & 396,3 & $-0,87$ \\
332 & 569,6 & 575,1 & $-0,97$ \\
446 & 765,2 & 772,4 & $-0,94$ \\
\hline
\end{tabular}

Fonte: (PRÓPRIOS AUTORES (2020)) 
Tabela 4: Comparação entre os momentos fletores no meio do vão: SAP2000 (1) e Ftool (2) - Veículo 2 S2

\begin{tabular}{c|c|c|c}
\hline Carga total (kN) & SAP2000 (kN.m) & Ftool (kN.m) & Variação entre os esforços por (1) e (2) (\%) \\
\hline 22,3 & 26,4 & 25,8 & 2,27 \\
190 & 224,9 & 218,9 & 2,67 \\
309 & 365,8 & 355,5 & 2,82 \\
376 & 445,1 & 432,8 & 2,76 \\
446 & 527,9 & 513,3 & 2,77 \\
\hline
\end{tabular}

Fonte: (PRÓPRIOS AUTORES (2020))

Tabela 5: Comparação entre os momentos fletores no meio do vão: SAP2000 (1) e Ftool (2) - Veículo 2S3

\begin{tabular}{c|c|c|c}
\hline Carga total $(\mathrm{kN})$ & SAP2000 (kN.m) & Ftool (kN.m) & Variação entre os esforços por (1) e (2) (\%) \\
\hline 22,3 & 32,6 & 32,7 & $-0,31$ \\
158 & 231,2 & 232,3 & $-0,48$ \\
386 & 564,7 & 568,5 & $-0,67$ \\
490 & 716,9 & 721,4 & $-0,63$ \\
549 & 803,2 & 808,1 & $-0,61$ \\
\hline
\end{tabular}

Fonte: (PRÓPRIOS AUTORES (2020))

A tabela 6 apresenta as variações médias para cada tipo de veículo.

Tabela 6: Variações médias entre os resultados do SAP2000 e do Ftool

\begin{tabular}{c|c|c|c|c|c}
\hline Veículo & O3C & 2C & $3 S$ & 2S2 & $2 S 3$ \\
\hline Variação (\%) & $-1,01$ & 2,42 & 0,17 & 2,66 & $-0,54$ \\
\hline
\end{tabular}

Fonte: (PRÓPRIOS AUTORES (2020))

Analisando-se os resultados encontrados, observa-se que todos apresentam variações percentuais pequenas, variando, em módulo, de 0,17 a 2,66\%. As diferenças encontradas podem se justificar nas diferentes abordagens adotadas por cada software e em possíveis arredondamentos realizados.

Frente a isso, o Ftool mostrou-se eficaz e confiável para ser utilizado na determinação de momentos fletores devido a cargas móveis em pontes compostas por 2 vigas biapoiadas.

\section{EXEMPLO NUMÉRICO 02}

O exemplo numérico 02 é baseado na ponte estudada por Trentini e Martins (2015). Os autores determinaram os esforços internos cortante e os momento fletor devido às cargas móveis do veículo normativo TB 450, da NBR 7188 (ABNT, 2013), sendo as cargas distribuídas p de $5 \mathrm{kN} / \mathrm{m}^{2}$ e as cargas pontuais $\mathrm{P}$ de $60 \mathrm{kN}$ cada. A ponte estudada pelos autores é de concreto protendido, com 35,00 m de comprimento e 16,10 m de extensão da seção transversal, a qual é composta por sete vigas, com $f_{c k}$ de $35 \mathrm{MPa}$. A figura 7 apresenta a seção transversal da ponte e a seção transversal da viga 1 , que é aquela a ser analisada.

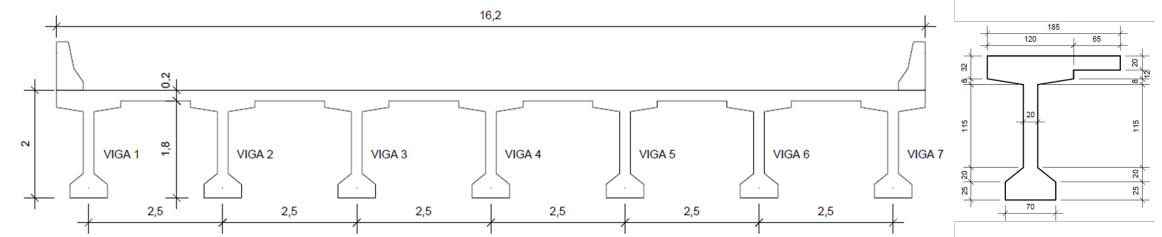

Figura 7: Perfis transversais da ponte e da viga 1 (TRENTINI, MARTINS, 2015) 
Os autores mencionados utilizaram o software de elementos finitos SAP2000 para determinar os valores máximos do esforço cortante e momento fletor na viga 1. Logo, os resultados encontrados através da modelagem no Ftool (2D) são comparados com aqueles obtidos por Trentini e Martins (2015), através da análise 3D da ponte. Nas análises realizadas no Ftool, o veículo é posicionado da mesma que o adotado por Trentini e Martins (2015), conforme mostrado na figura 8.

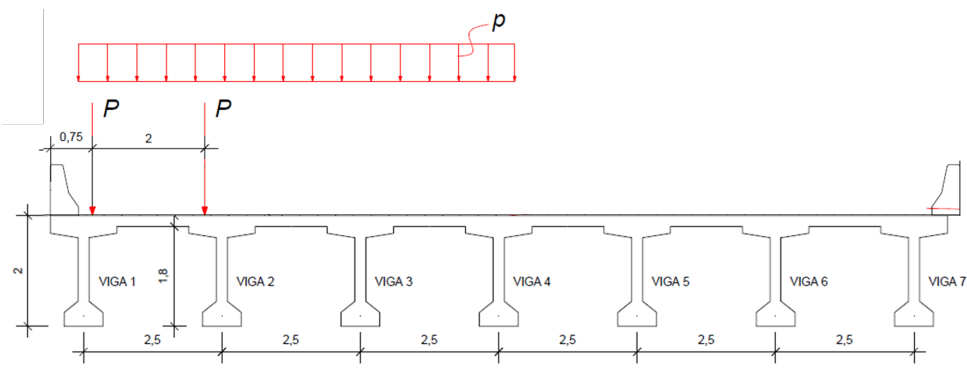

Figura 8: Esquema de posicionamento do trem tipo (TRENTINI, MARTINS (2015))

Além de ser um software pago, o que limita sua utilização por estudantes, professores e pesquisadores, conforme colocado pelos autores, é o fato da modelagem da ponte feita no SAP2000 requer uma série de etapas e procedimentos que demandam conhecimento profundo sobre a utilização do software, atenção e tempo para se modelar. Além disso, uma vez modelada, deve-se considerar, também, o tempo que o programa levará para o processamento da estrutura.

Ademais, para se empregar a modelagem no Ftool, por se tratar de uma ponte com vigas múltiplas é aplicado o Processo de Fauchart. Moura et al. (2016, p. 2) pontuam que o Método de Fauchart é "um procedimento de cálculo simples cuja reconhecida eficácia permite transformar o problema bidimensional em séries unidimensionais" (MOURA et al., 2016, p. 2).

Esse processo propõe que se calcule uma estrutura plana de forma que ela represente uma faixa unitária de laje e as vigas são substituídas por molas de forma a impedir a rotação e o deslocamento vertical dos respectivos pontos. A figura 9 mostra a seção transversal da ponte analisada modelada no Ftool considerando-se os princípios do Processo de Fauchart.

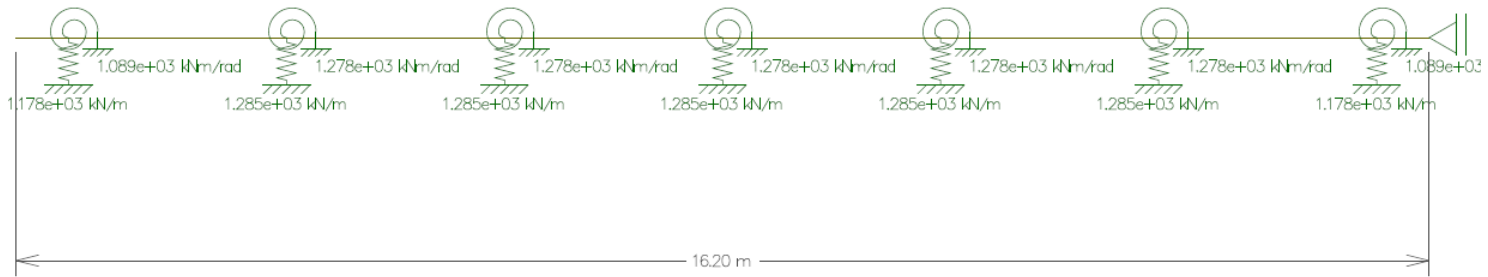

Figura 9: Seção transversal pelo processo de Fauchart do Exemplo Numérico 02 (PRÓPRIOS AUTORES (2020))

Trentini e Martins (2015) precisaram criar uma vasta malha de elementos finitos com 29.808 elementos sólidos. A figura 10 apresenta a modelagem 3D no SAP2000. 


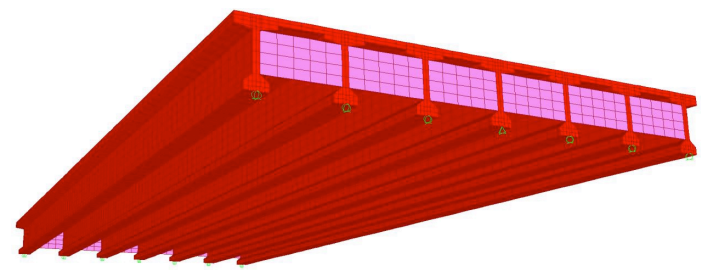

Figura 10: Modelagem no SAP2000 do Exemplo Numérico 02 (TRENTINI, MARTINS (2015))

A modelagem, no Ftool, foi muito mais simples, podendo ser separada em duas etapas. Primeiramente, criou-se um arquivo no qual se fez a modelagem 2D da seção transversal da ponte, onde as vigas foram representadas pelas molas acima explicadas, além da atribuição das propriedades dos materiais e geométricas. Em seguida, criou-se um novo arquivo no qual ocorreu a modelagem da viga analisada, aplicação das propriedades dos materiais e geométricas, e, por fim, a aplicação da carga móvel do TTL a ser analisada. Nesse direcionamento, a figura 11 apresenta a viga 1 modela no Ftool.

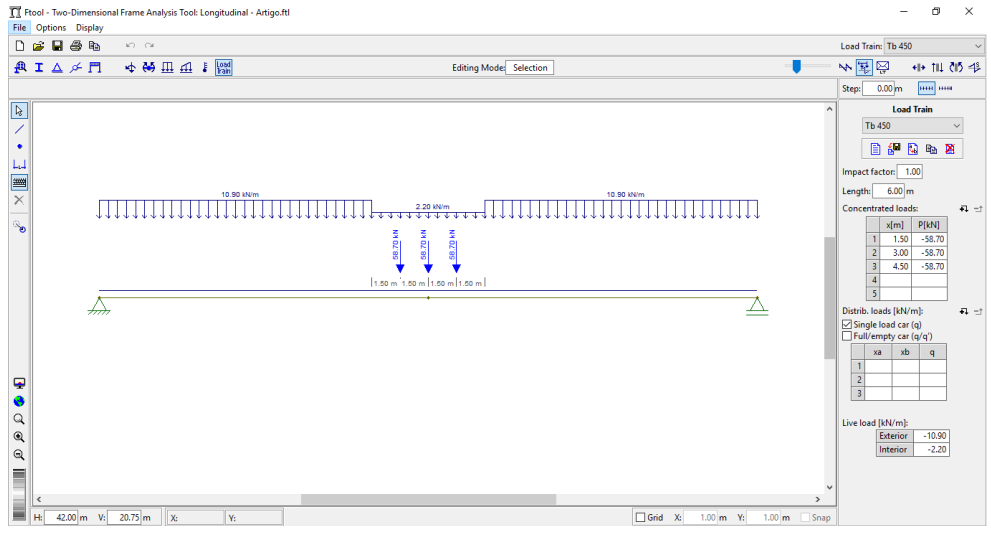

Figura 11: Modelagem da viga 01 do Exemplo Numérico 02 no Ftool (PRÓPRIOS AUTORES (2020))

\subsection{Análise e discussão dos resultados do Exemplo Numérico 02}

Os resultados encontrados para ambas as abordagens são apresentados na tabela 7, bem como a variação encontrada entre ambos os resultados.

Tabela 7: Comparação entre os esforços no meio do vão: SAP2000 (1) e Ftool (2) - Exemplo Numérico 02

\begin{tabular}{c|c|c|c}
\hline Análise & SAP2000 & Ftool & $\begin{array}{c}\text { Variação entre os esforços pelos } \\
\text { programas (1) e (2) (\%) }\end{array}$ \\
\hline Esforço cortante máximo (kN) & 328,48 & 327,67 & 0,25 \\
Momento fletor máximo (kN.m) & $2.705,48$ & $2.704,29$ & 0,04 \\
\hline
\end{tabular}

Fonte: (PRÓPRIOS AUTORES (2020))

Consoante a tabela acima, verifica-se que o esforço cortante e o momento fletor máximos obtidos pelo Ftool são muito próximos àqueles resultantes do SAP2000, com variações inferiores a 1\%. Portanto, empregando-se o Ftool, associado ao Processo de Fauchart, observa-se que eles são mais simples na questão dos cálculos, da modelagem e do tempo de processamento comparado à modelagem em elementos finitos, através do SAP2000, realizada pelos autores. 
Ademais, a utilização e modelagem no Ftool não requereu conhecimentos avançados, e foram necessários aproximadamente 20 nós por arquivo, para o modelo, e processamento praticamente instantâneo. Por fim, pelo Ftool ser gratuito, nesta ponte analisada, em termos de modelagem, processamento e proximidade de resultados, apresenta-se como software indicado.

\section{EXEMPLO NUMÉRICO 03}

O exemplo numérico 03 utiliza a ponte estudada por Souza (2015). A ponte analisada pelo autor é de concreto, com 30,00 m de comprimento e com 16,00 m de extensão da seção transversal, a qual é composta por três vigas, com $f_{c k}$ de $40 \mathrm{MPa}$. A figura 12 apresenta a ponte vista de cima e a seção transversal da viga 1, que será aquela analisada.

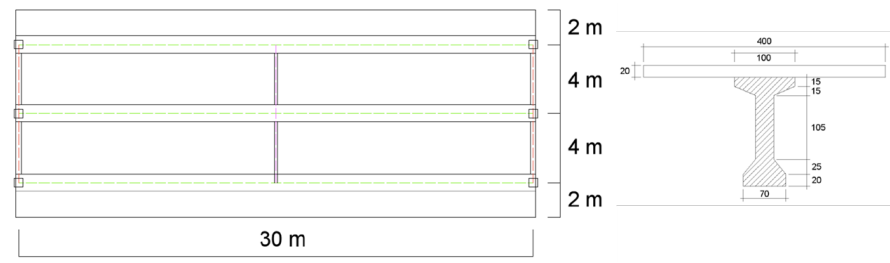

Figura 12: Vista superior da ponte perfil transversal da viga 1 (SOUZA (2015))

A ponte é modelada no Ftool (2D) e os resultados encontrados são comparados com aqueles obtidos por Souza (2015), que empregou o método de analogia de grelha para a modelagem, utilizando o "programa GAP-GEL $v 1$, baseado no método dos deslocamentos, desenvolvido pelo professor Daniel Domingues Loriggio, Dr." (SOUZA, 2015, p. 101).

Segundo o autor, "os elementos longitudinais foram divididos em elementos reticulares de um metro de vão, e os elementos transversais, em elementos reticulares unitários, enumerados de esquerda à direita de cima para baixo" (SOUZA, 2015, p. 107), resultando em cerca de 100 elementos. Já no modelo do Ftool foram necessários apenas 5 nós ao longo da seção transversal.

Mais uma vez, chama-se atenção para a necessidade de se conhecer bem o funcionamento e a utilização do software GAP-GEL $v 1$, bem como os diversos procedimentos e etapas requeridos na modelagem da ponte, bem como no tempo de processamento.

Novamente emprega-se o Processo de Fauchart associado à modelagem no Ftool por te tratar de uma ponte com três vigas. O desenvolvimento e aplicação do Processo de Fauchart seguem os mesmos parâmetros, conforme explicado no Exemplo Numérico 02.

\subsection{Análise e discussão dos resultados do Exemplo Numérico 03}

São comparados os resultados para os valores máximos de momentos fletores para uma carga unitária em três pontos do comprimento da viga, em 0,00, 5,00, 10,00 e 15,00 m. Os resultados encontrados para ambas as abordagens são apresentados na tabela 8 , bem como a variação encontrada entre ambos os resultados. 
Tabela 8: Comparação entre os momentos fletores máximos: GAP-GEL v1 (1) e Ftool (2) - Exemplo Numérico 03

\begin{tabular}{c|c|c|c}
\hline $\begin{array}{c}\text { Ponto de análise ao longo da } \\
\text { viga }(\mathrm{m})\end{array}$ & \multicolumn{2}{|c|}{ Momento fletor máximo (kN.m) } & $\begin{array}{c}\text { Variação entre os esforços pelos } \\
\text { programas (1) e (2) (\%) }\end{array}$ \\
\cline { 2 - 3 } & GAP-GEL v1 & Ftool & 0,00 \\
5,00 & 0,0 & 0,0 & 8,11 \\
5,00 & 3,7 & 3,4 & 3,51 \\
10,00 & 5,7 & 5,5 & 3,17 \\
15,00 & 6,3 & 6,1 & \\
\hline
\end{tabular}

Fonte: (PRÓPRIOS AUTORES (2020))

Os valores encontrados pelo Ftool apresentam variação inferior a 9\%, sendo a média das variações de aproximadamente $3,70 \%$. Com isso, os valores apresentados pelo Ftool podem ser tidos como aceitáveis e confiáveis. Ademais, observa-se que os resultados para os pontos 10,00 e $15,00 \mathrm{~m}$ estão próximos à média, sendo apenas o resultado para ponto $5,00 \mathrm{~m}$ aquele que se destoou mais. Isso pode ter ocorrido devido a alguns fatores, como algum arredondamento ou consideração diferente das demais na modelagem para esse ponto feita por Souza (2015). Em suma, o Ftool, novamente, apresentou-se como uma ferramenta confiável e robusta.

\section{EXEMPLO NUMÉRICO 04}

O exemplo numérico 04 é baseado no modelo de ponte concebido por Giardin (2019). Neste exemplo são analisados os valores das cargas máximas do TB 450, de acordo com a figura 13 , colocados na posição mais crítica para a viga 1. Com isso, considerando-se o trem tipo na posição que leva a maior reação da viga estudada chega-se ao conjunto de cargas do trem-tipo longitudinal (TTL). É justamente esse conjunto de cargas do TTL que são comparadas através dos resultados obtidos por Giardin (2019), com a utilização do Ansys, na versão Mechanical APDL 16.1 Academic.

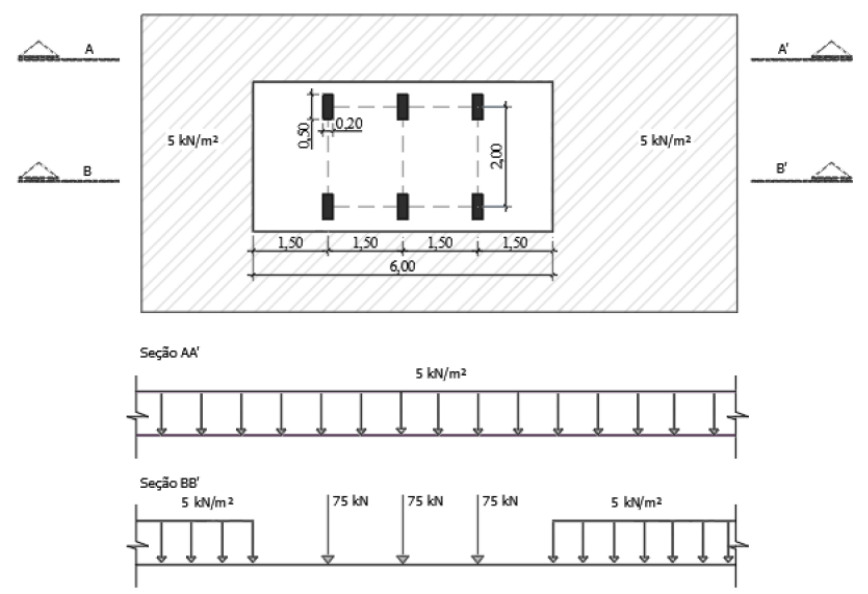

Figura 13: Trem tipo TB 450 (GIARDIN (2019))

A ponte estudada por Giardin (2019) é pré-moldada composta por oito vigas em concreto armado, com 10,00 m de vão e 8,0 0m de extensão na seção transversal. A autora utilizou concreto com $f_{c k}$ de $40 \mathrm{MPa}$, e agregado da mistura basalto, com $\alpha_{\mathrm{E}}$ de 1,2. A figura 14 mostra a seção transversal da ponte analisada. 


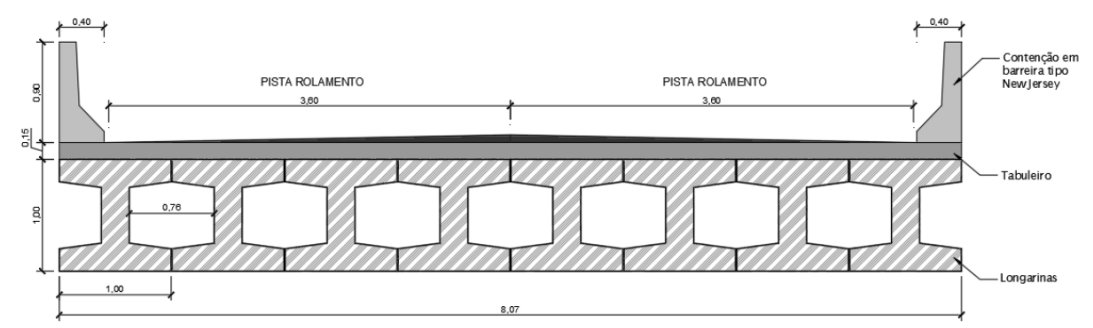

Figura 14: Seção transversal da ponte do Exemplo Numérico 04 (GIARDIN (2019))

Nas análises realizadas no Ftool, o veículo é posicionado da mesma que o adotado por Giardin (2019), conforme mostrado na figura 15.

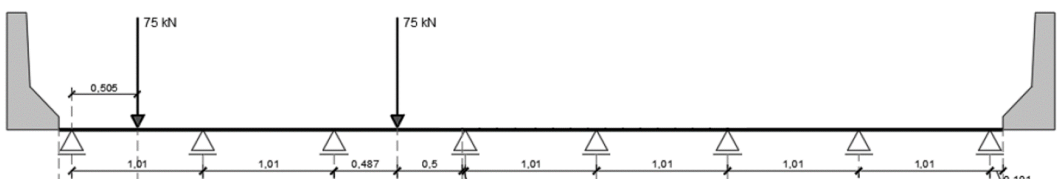

Figura 15: Posição do TB 450 do Exemplo Numérico 04 (GIARDIN (2019))

Assim como no caso dos demais exemplos numéricos, a modelagem 3D no Ansys requer conhecimentos mais densos sobre seu funcionamento e utilização, bem como elevado tempo de modelagem e processamento da estrutura, tão mais longo, quanto maiores forem os números de elementos sólidos (de malhas) criados. A figura 16 apresenta a modelagem 3D feita pela autora e a figura 17 a modelagem feita no Ftool para a viga 1.

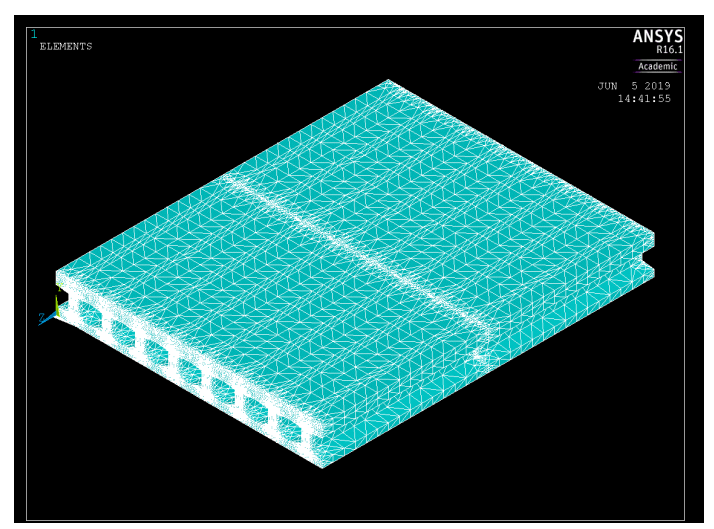

Figura 16: Modelagem no software Ansys do Exemplo numérico 04 (GIARDINI (2019))

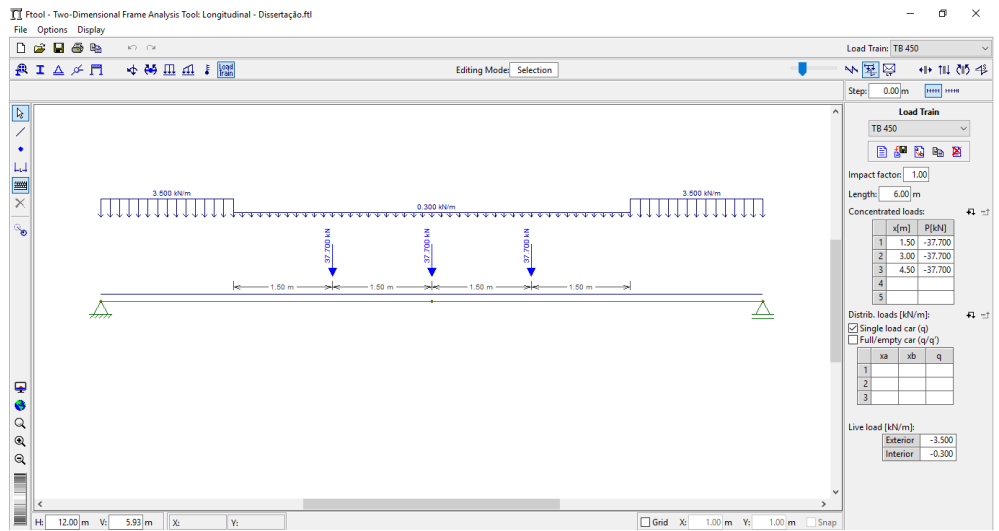

Figura 17: Modelagem no Ftool da viga 1 Exemplo numérico 04 (PRÓPRIOS AUTORES, 2020) 


\subsection{Análise e discussão dos resultados do Exemplo Numérico 04}

No que tange à análise e discussão sobre os resultados encontrados para ambas as análises,convém mencionar que se encontram na tabela 9. A tabela apresenta as diferentes cargas obtidas a partir do TB 450 que compõem o TTL máximo para a viga 1, determinados pelo Ansys (1) e pelo Ftool (2) e a variação percentual encontrada entre ambos os resultados. As porcentagens negativas indicam que os valores determinados pelo Ftool são maiores que os encontrados pelo Ansys.

Tabela 9: Comparação entre as cargas do TTL máximo: Ansys (1) e Ftool (2) - Exemplo Numérico 04

\begin{tabular}{c|c|c|c}
\hline \multirow{2}{*}{ Carga } & \multicolumn{2}{|c|}{ Software de análise } & \multicolumn{2}{c}{$\begin{array}{c}\text { Variação entre os esforços } \\
\text { pelos programas (1) e (2) (\%) }\end{array}$} \\
\cline { 2 - 3 } & Ansys & Ftool & 5,70 \\
Pontual $(\mathrm{kN})$ & 39,975 & 37,695 & $-1,95$ \\
Distribuída interna $(\mathrm{kN} / \mathrm{m})$ & 0,333 & 0,339 & $-0,08$ \\
Distribuída externa $(\mathrm{kN} / \mathrm{m})$ & 3,795 & 3,798 & \\
\hline
\end{tabular}

Fonte: (PRÓPRIOS AUTORES (2020))

Nesse exemplo, empregou-se outro software de elementos finitos, o Ansys, e mesmo assim, com base nos resultados, verifica-se que eles, em média, a variação foi inferior a 2,60\%, sendo o valor encontrado para a carga pontual, pelo Ansys, 5,70\% maior que aquele encontrado pelo Ftool. Portanto, dessa forma, constata-se, mais uma vez, que o Ftool é indicado para a análise de cargas móveis em vigas de pontes e/ou viadutos.

\section{CONSIDERAÇÕES FINAIS}

Pontes e viadutos são elementos essenciais em uma infraestrutura de transportes. Nesse sentido, é importante o desenvolvimento de pesquisa acerca dessas estruturas. Para tanto, em muitos casos, faz-se necessário o emprego de softwares pagos, para que se realize a análise estrutural de cargas móveis em vigas de pontes e/ou viadutos.

Com isso, este trabalho teve como foco principal evidenciar a aplicabilidade, robustez e confiabilidade do Ftool nas análises estruturais de cargas móveis em vigas de pontes com diferentes números de vigas. Seja para determinação das cargas que compõem o trem tipo longitudinal máximo, dos esforços cortes e das envoltórias de momentos fletores de pontes e/ou viadutos.

A escolha pela utilização do Ftool deve-se ao fato de ser uma ferramenta gratuita e por apresentar modelagem muito mais simples, não requerer conhecimentos prévios complexos e densos para sua utilização e por apresentar tempo de processamento praticamente instantâneo, ao contrário dos softwares apresentados.

Em todos os quatro exemplos numéricos apresentados, as diferenças encontradas entre os resultados apresentados pelos softwares pagos, SAP2000, Ansys e GAP-GEL v1, foram inferiores a $9 \%$ no pior dos casos, mas na maioria deles, essa variação foi inferior a $3 \%$.

Essas diferenças podem ser tidas como aceitáveis, pois, em etapas de análise e dimensionamento posteriores há outros fatores de cálculo a serem considerados, bem como são aplicados diferentes coeficientes de majoração normativos, o que faz com que essa diferença ou se dilua ao longo das etapas seguintes, ou seja insignificante. 
Diante o exposto, conclui-se, portanto, que o Ftool se mostrou eficaz e robusto, com resultados confiáveis e, por isso é recomendado para a determinação dos diferentes esforços e TTL nas vigas, devido a diferentes cargas móveis atuantes em tabuleiros de pontes compostas por duas, três, sete e oito vigas. Dessa maneira, não ocorre a necessidade de se empregar software mais complexos e pagos para se realizar tais análises.

\subsection{Agradecimentos}

Faz-se especial agradecimento à Coordenação de Aperfeiçoamento de Pessoal de Nível Superior CAPES, que é uma fundação vinculada ao Ministério da Educação do Brasil, pela bolsa de estudos concedida ao autor correspondente. O presente trabalho foi realizado com apoio da CAPES Código de Financiamento 88882.426436/2019-01.

\section{REFERÊNCIAS}

Abrishami, H. (2015). Design of Reinforced Concrete Bridges. University of Toronto. Recuperado de http://civmin.utoronto.ca/wp-content/uploads/2015/09/CIV498 2017 Group-2 ReportPart-A.pdf

Albuquerque, I. M. de. (2012). Determinação da Vida Útil à Fadiga em Ponte de Concreto Armado Considerando o Espectro de Veículos Reais. (Trabalho de conclusão de curso). Departamento de Estruturas da Escola Politécnica da Universidade Federal do Rio de Janeiro, Rio de Janeiro, Brasil.

ABNT NBR 7188, de 11 de novembro de 2013. Carga Móvel Rodoviária e de Pedestres em Pontes, Viadutos, Passarelas e outras estruturas. Rio de Janeiro: Associação Brasileira de Normas Técnicas.

Barros, M. A., Silva, L. G. S., Souza, A. L. A. P, Crispim, E. C. S. (2018, setembro). Sistemas mecânicos treliçados para auxiliar o aprendizado prático em mecânica geral. Anais do Congresso Brasileiro de Educação em Engenharia, Salvador, BA, Brasil, 46. Recuperado de http://www.abenge.org.br/sis artigos.php

Carvalho, R. C. (2017). Introdução ao estudo de pontes. Universidade Federal de São Carlos. São Carlos, SP, Brasil.

Carvalho Neto, J. A., Veloso, L. A. C. M. (2014, maio). Algoritmo para Análise Estrutural de Pontes Submetidas a Cargas Móveis. Anais do Congresso Brasileiro de Pontes e Estruturas, Rio de Janeiro, RJ, Brasil, 8. Recuperado de http://www.abpe.org.br/trabalhos/trab 69.pdf

Cruz Santos, J. P. M. (2016). Análise da carga móvel e da protensão na superestrutura de pontes biapoiadas em vigas pré-moldadas protendidas (Trabalho de conclusão de curso). Escola Politécnica, Universidade Federal do Rio de Janeiro, RJ, Brasil.

Drun, A. G., Souza, R. A. (2018, maio). Comparação Entre os Veículos de Carga Atuais e o Trem-Tipo Recomendado Pela NBR 7188. Anais do Congresso Brasileiro de Pontes e Estruturas, Rio de Janeiro, RJ, Brasil, 10. Recuperado de http://www.abpe.org.br/trabalhos2018/153.pdf 
Ftool. (2018). Um Programa Gráfico-Interativo para Ensino de Comportamento de Estruturas. Versão Educacional 4.00, Rio de Janeiro, RJ, Brasil. Recuperado de https://www.ftool.com.br/Ftool/site/about

Ftool. (2019). A Graphical-Interactive Program for Teaching Structural Behavior. Recuperado de https://www.ftool.com.br/Ftool/

Giardin, L. H. F. (2019). ANÁLISE DE PONTES RODOVIÁRIAS DOTADAS DE MÚLTIPLAS VIGAS EM CONCRETO PRÉ-MOLDADO COM SEÇÃO DUPLO T SERVINDO DE LAJE. (Trabalho de conclusão de curso). Departamento de Engenharia Civil da Universidade Santa Cruz do Sul, RS, Brasil.

Martha, L. F. (2018). Manual do Ftool: Programa Gráfico-Interativo para Análise de Estruturas, versão 4.00, Rio de Janeiro, RJ, Brasil.

Mascarenhas, F. J. R., Dias, V. C., Bonaldo, E. (2016, setembro). Impactos da utilização dos softwares no processo de ensino-aprendizagem na disciplina de teoria de estruturas na PUC Minas Barreiro. Anais do Congresso Brasileiro de Educação em Engenharia, Natal, RN, Brasil, 44. Recuperado de http://www.abenge.org.br/cobenge/arquivos/3/anais/anais/161219.pdf

Mascarenhas, F. J. R, Chust Carvalho, R. (2019). Vida útil à fadiga da armadura longitudinal de vigas de pontes de concreto armado frente ao tráfego real de veículos pesados. Revista ALCONPAT, 9(3), 303-319. https://doi.org/10.21041/ra.v9i3.375

Mascarenhas, F. J. R., Carvalho, R. C., Vitório, J. A. P. (2019, outubro). Uma análise das atuais condições das pontes e viadutos das rodovias brasileiras. Anais do Congresso Brasileiro do Concreto, Fortaleza, CE, Brasil, 61. Recuperado de http://ibracon.org.br/eventos/61cbc/artigos/61cbc/artigos

Medeiros, C. A. (2016, maio) Análise de Fadiga para uma Viga de Rolamento de Ponte Rolante. Anais do Congresso Brasileiro de Pontes e Estruturas, Rio de Janeiro, RJ, Brasil, 9. Recuperado de http://www.abpe.org.br/trabalhos2016/35.pdf

Milani, C. J., KRIPKA, M. (2012). Diagnosis of Pathologies in Bridges of the Road System in Brazil. CONSTRUCTII, 1, 26-34.

Moura, M. W., Ferreira, M. W., Real, M. V., Santos, G. C. (2016, maio). Comparação do Método de Fauchart e do Método dos Elementos Finitos na Avaliação da Distribuição de Esforços Transversais em Pontes Rodoviárias Anais do Congresso Brasileiro de Pontes e Estruturas, Rio de Janeiro, RJ, Brasil, 9. Recuperado de http://www.abpe.org.br/trabalhos2016/61.pdf

Passos, D. S., Venega, V. S., Rocha, M. L. (2017). SOFTWARES PARA SUPORTE NO ENSINO DE ENGENHARIA CIVIL: UM MAPEAMENTO SISTEMÁTICO DO USO NAS INSTITUIÇÕES BRASILEIRAS. Revista Cereus, 9(4), 2-18. 10.18605/2175-7275/cereus.v9nep2-18

Rodrigues, I. M., Colmenero, J. C. (2009, outubro). A importância da manutenção das rodovias para o sistema de redes logísticas. Anais do ENCONTRO NACIONAL DE ENGENHARIA DE PRODUÇÃO, Salvador, BA, Brasil, $29 . \quad$ Recuperado de http://www.abepro.org.br/biblioteca/enegep2009 TN STO 097659 14532.pdf 
Rota Oeste S.A. (2016). Análise do impacto do tráfego na vida útil das OAE's - BR $163 \mathrm{KM} 0$ ao KM 855. Relatório técnico à Agência Nacional de Transportes Terrestres. Recuperado de http://www.antt.gov.br/rodovias/Concessoes Rodoviarias/Rota do Oeste/CRO Relatorio de Pesquisa RDT.html

Santos JR, E., Barbosa, J. R. A., Lima, W. N., Rodrigues Filho, J. M. (2018, setembro). Metodologias ativas aplicadas em resistência dos materiais para engenharia civil: projeto estrutural metálico simplificado usando recurso computacional. Anais do Congresso Brasileiro de Educação em Engenharia, Salvador, BA, Brasil, 46. http://www.abenge.org.br/sis artigos.php

Silva, E. C. N., Stoeterau, R. L. (2016). Introdução ao MEF: Notas de Aula. Escola Politécnica, Departamento de Engenharia Mecatrônica e de Sistemas Mecânicos da Universidade de São Paulo, São Paulo, SP, Brasil. Recuperado de http://sites.poli.usp.br/d/PMR5222/MEFtutorial.pdf

Souza, C. F. P. (2015). ANÁLISE DA DISTRIBUIÇÃO DE MOMENTOS FLETORES EM PONTES BIAPOIADAS (Dissertação de Mestrado). Programa de Pós-Graduação em Engenharia Civil da Universidade Federal de Santa Catarina, Florianópolis, SC, Brasil. Recuperado de https://repositorio.ufsc.br/handle/123456789/134663

Trentini, E. V. W., Martins, C. H. (2015). CÁLCULO DOS ESFORÇOS ATUANTES EM LONGARINAS DE VIADUTOS OU PONTES UTILIZANDO O PROCESSO DE FAUCHART E ANÁLISE POR MÉTODO DOS ELEMENTOS FINITOS. Revista de Engenharia e Tecnologia, 3(7), 162-173.

Turmina, G. (2016). Estudo dos esforços atuantes em uma ponte em concreto armado (Trabalho de conclusão de curso). Curso de Engenharia Civil da Universidade Tecnológica Federal do Paraná. Pato Branco, PR, Brasil. Recuperado de http://repositorio.roca.utfpr.edu.br/ispui/bitstream/1/7665/1/PB COECl 20161 05.pdf 